\title{
Nonnecrotizing anterior scleritis mimicking orbital inflammatory disease
}

\author{
Michelle Chen Lynch' \\ Andrew B Mick ${ }^{2}$ \\ 'Optometry Clinic, Ocala West \\ Veterans Affairs Specialty Clinic, \\ Ocala, FL, USA; ${ }^{2}$ Eye Clinic, \\ San Francisco VA Medical Center, \\ San Francisco, CA, USA
}

This article was published in the following Dove Press journal:

Clinical Optometry

12 August 2013

Number of times this article has been viewed
Correspondence: Michelle Chen Lynch Optometry Clinic, Ocala West VA Specialty Clinic, 3307 SW 26th Ave, Ocala, FL 3447I, USA

Tel + I $35286 \mid 3940$ ext 1216

Fax + I 352 86I 394I

Email Michelle.Lynch@va.gov
Background: Anterior scleritis is an uncommon form of ocular inflammation, often associated with coexisting autoimmune disease. With early recognition and aggressive systemic therapy, prognosis for resolution is good. The diagnosis of underlying autoimmune disease involves a multidisciplinary approach.

Case report: A 42-year-old African American female presented to the Eye Clinic at the San Francisco Veteran Affairs Medical Center, with a tremendously painful left eye, worse on eye movement, with marked injection of conjunctiva. There was mild swelling of the upper eyelid. Visual acuity was unaffected, but there was a mild red cap desaturation. The posterior segment was unremarkable. The initial differential diagnoses included anterior scleritis and orbital inflammatory disease. Oral steroid treatment was initiated with rapid resolution over a few days. Orbital imaging was unremarkable, and extensive laboratory work-up was positive only for antinuclear antibodies. The patient was diagnosed with idiopathic diffuse, nonnecrotizing anterior scleritis and has been followed for over 5 years without recurrence. The rheumatology clinic monitors the patient closely, as suspicion remains for potential arthralgias including human leukocyte antigen-B27-associated arthritis, lupus-associated arthritis, seronegative rheumatoid arthritis, recurrent juvenile idiopathic arthritis, and scleroderma, based on her constitutional symptoms and clinical presentation, along with a positive anti-nuclear antibody lab result.

Conclusion: Untreated anterior scleritis can progress to formation of cataracts, glaucoma, uveitis, corneal melting, and posterior segment disease with significant risk of vision loss. Patients with anterior scleritis must be aggressively treated with systemic anti-inflammatories and monitored for recurrence of sight-threatening or life-threatening complications relating to systemic disease.

Keywords: anterior scleritis, nonnecrotizing scleritis, ocular inflammation, anti-inflammatory therapy, systemic steroids

\section{Introduction}

Scleritis is a painful and chronic inflammatory disorder of the sclera and episclera. It is often associated with underlying systemic disease and must be treated with systemic therapy. Depending on the extent and location of involvement, visual prognosis is variable. Inflammation of the sclera can be defined as anterior or posterior scleritis based on the clinical classification proposed by Watson and Hayreh. ${ }^{1}$ Anterior scleritis is categorized as either necrotizing or nonnecrotizing. Nonnecrotizing anterior scleritis is further subdivided as diffuse or nodular. Diffuse scleritis describes widespread enlargement of the episcleral vessels with distortion of the normal vascular pattern. Nodular scleritis consists of scleritis with either single or multiple elevated foci of inflammation. These nodules are nonmobile areas of scleral edema with dilated, deep 
episcleral vessels. ${ }^{2}$ The most serious form is necrotizing anterior scleritis, which demonstrates severe edema and intense dilation of the deep episcleral plexus and superficial vessels. This form of anterior scleritis carries the greatest risk of scleral perforation and vision loss. ${ }^{2}$ The anterior portion of the sclera is involved in $85 \%-90 \%$ of scleritis. ${ }^{3}$ Posterior scleritis is scleral inflammation confined to the posterior segment and may be accompanied by a serous detachment and subretinal exudates. Posterior scleritis can present in isolation or with an accompanying anterior scleritis. ${ }^{4}$ It is critical to differentiate scleritis from other ocular entities in order to initiate appropriate anti-inflammatory treatment in a timely manner before vision-threatening complications occur.

\section{Case report}

A 42-year-old African American female presented to the Eye Clinic at the San Francisco Veteran Affairs Medical Center, complaining of pain, redness, and photophobia in her left eye that began the night before. She noted tenderness upon palpation of her slightly edematous eyelid that prohibited her from fully opening her eye. Medical history was significant for gastroesophageal reflux disorder, stomach ulcers, iron deficiency anemia, depression, dysmenorrhea, spinal stenosis, and degenerative disc disease. She also had a self-reported diagnosis of juvenile rheumatoid arthritis (now commonly referred to as juvenile idiopathic arthritis), which involved the joints of her wrists, knees, shoulders, and hands. She had taken oral steroids in the past to help minimize the inflammation and could not recall any previous history or treatment for ocular inflammation.

Corrected visual acuity was 20/20 in each eye. Pupils were round and reactive to light with no afferent pupillary defect. The patient reported positive desaturation on red cap testing of the left eye, approximately $70 \%$ compared to $100 \%$ of the right eye. Extraocular motility was smooth and unrestricted with moderate to severe pain reported upon movement in the left eye, but the patient denied diplopia symptoms. Confrontation visual fields were full to finger count, and color testing with Ishihara plates was normal in each eye. Intraocular pressure was $23 \mathrm{mmHg}$ in each eye by Goldmann applanation tonometry.

Under gross examination, the superior conjunctiva in the left eye appeared elevated, with a markedly bright red coloration and purplish hue (Figure 1A and B). The conjunctival involvement extended temporally and inferiorly but was not as intense as superiorly. Slit lamp examination showed a diffusely meaty, deep red colored conjunctival tissue that persisted to the level of the sclera. The episcleral
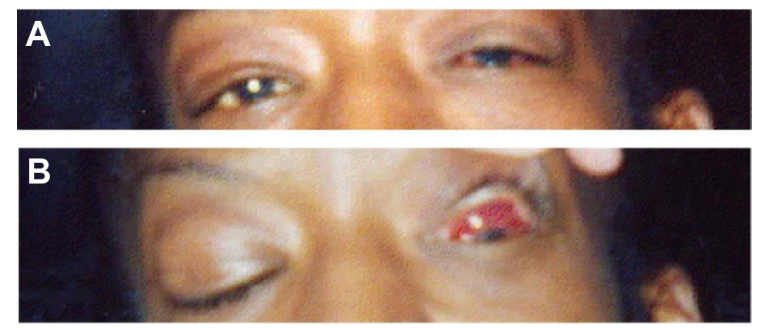

Figure I Gross examination on the day of presentation.

Notes: Note the left lid edema and ptosis in primary gaze $(\mathbf{A})$ and the marked redness and edema of the superior sclera and episclera (after application of $2.5 \%$ phenylephrine) (B).

edema was greater superiorly and associated with marked dilation and elevation of the episcleral vessels. The details of the underlying sclera were not visible through the dark red conjunctiva. The conjunctival vessels did not blanch with the application of $2.5 \%$ phenylephrine ophthalmic solution. The cornea was clear without opacification or signs of thinning. No anterior chamber or vitreous reaction was noted. The lens had a few punctate cortical opacities. Ocular fundus examination was unremarkable with no observable elevation of the retina. B-scan ultrasonography of the left eye confirmed that there was no posterior scleral thickening or retinal detachment (Figure 2). The right eye was unremarkable.

Review of systems was negative for thyroid- or endocrinerelated disorders, recent infections, sinusitis, gastrointestinal discomfort, and weakness of the extremities. Laboratory work-up included complete blood count, Westergren erythrocyte sedimentation rate, $\mathrm{C}$-reactive protein, reactive plasma reagin, rheumatoid factor, anti-nuclear antibody (ANA), and anti-cytoplasmic antibody. The results of the work-up are shown in Table 1. Of note, there was a mildly elevated ANA, and the rheumatoid factor was nonreactive.

Magnetic resonance imaging (MRI) was ordered to rule out an acute optic perineuritis, which can occur in the presence

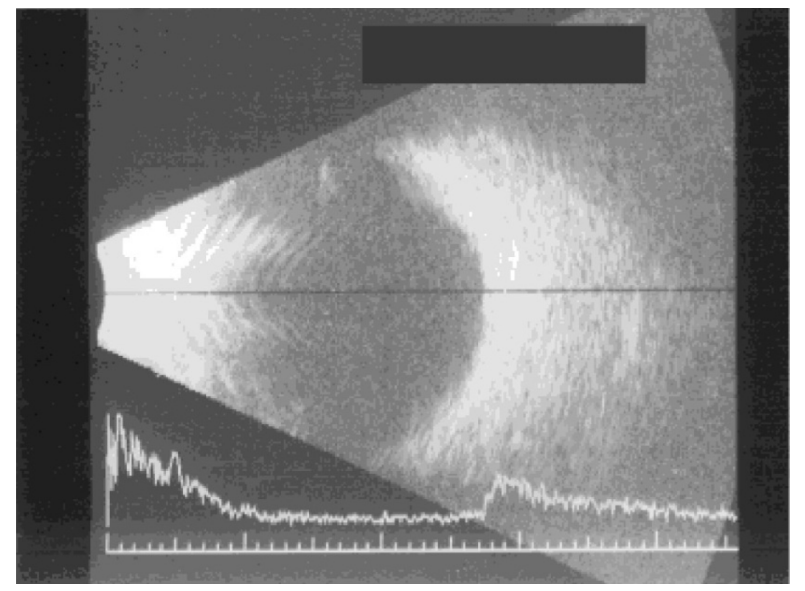

Figure 2 B-scan ultrasonography of left eye without evidence of posterior scleritis. 
Table I Patient's lab test results

\begin{tabular}{lll}
\hline Lab tests & Results & Reference range \\
\hline Complete blood count & & \\
- White blood cells & $3.20 \mathrm{k} / \mathrm{cmm}$ & $4.8-10.8$ \\
- Neutrophils (absolute) & $1.94 \mathrm{k} / \mathrm{mcL}$ & $2.2-8.6$ \\
ESR (Westergren) & $25 \mathrm{~mm} / \mathrm{hour}$ & $0-20$ \\
CRP & $<5 \mathrm{mg} / \mathrm{L}$ & $0-5$ \\
RPR (serum) & Nonreactive & \\
Rheumatoid factor & Negative & \\
ANA & $15 \mathrm{IU} / \mathrm{mL}$ & $0-7.5$ \\
ANA (titer calculated) & $\mathrm{I}: 160 \mathrm{nucleolar}$ & $<\mathrm{I:40}$ \\
ANCA IFA & $<\mathrm{I:I6}$ & $<\mathrm{I:16}$ \\
\hline
\end{tabular}

Abbreviations: ANA, anti-nuclear antibody; ANCA IFA, anti-cytoplasmic antibody immunofluorescence assay; CRP, C-reactive protein; ESR, erythrocyte sedimentation rate; RPR, reactive plasma reagin.

of a positive red cap test without an afferent pupillary defect. Optic perineuritis is a form of orbital inflammatory disease that involves inflammation of the optic nerve sheath and can be mistaken for demyelinating optic neuritis due to overlapping symptoms and clinic presentation. Symptoms of optic perineuritis include a sudden decline in vision, pain with eye movement, and either a swollen or normal appearing optic nerve. Diagnosis is confirmed by a combination of clinical and radiologic findings, which include perineural enhancement and "streaky" orbital fat that may or may not have extraocular muscle involvement on MRI. Unlike demyelinating optic neuritis, optic perineuritis is responsive to oral steroids. ${ }^{5}$ The patient was started on $60 \mathrm{mg}$ of oral prednisone daily, proactively for 4 days during the time of her first presentation to the clinic, followed by a 3-week taper. She was able to undergo MRI on the same day of her eye examination, but the results were not immediately available. The differential diagnoses were orbital inflammatory disease versus diffuse anterior scleritis, both of which are responsive to oral steroids. Also, she had a reported history of stomach ulcers so even though oral nonsteroidal anti-inflammatory drugs (NSAIDs) were a consideration if this were scleritis, the patient did not tolerate them well. She previously had a good response to oral steroids for intermittent inflammation in her joints. She denied any previous infectious conditions, including herpes, syphilis, and tuberculosis, and her history was not high risk. Sarcoid was also ruled out because of her longstanding history of joint inflammation with joint pains since childhood.

The interpretation of the MRI by the radiology clinic, which was received the following day, indicated that there was no evidence of thickening of the extraocular muscles, orbital fat, or enhancement of the optic nerve to suggest orbital inflammatory disease (Figure 3). The patient was diagnosed with diffuse nonnecrotizing anterior scleritis. The patient returned

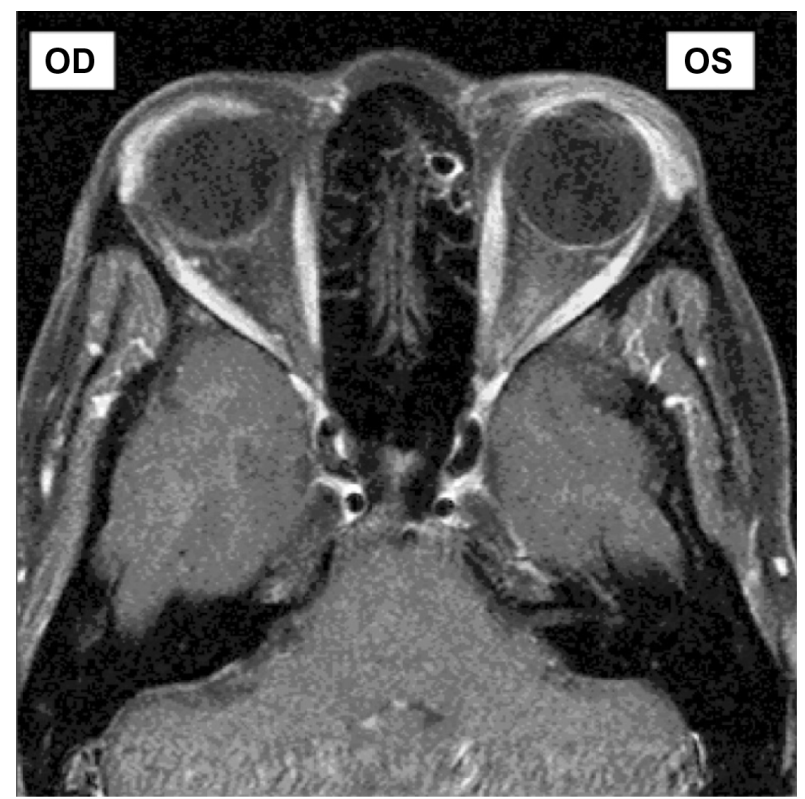

Figure 3 Axial view of T2 weighted magnetic resonance imaging.

Notes: No enhancement of optic nerve or involvement of extraocular muscles or orbital fat in the left eye. Mild anterior globe enhancement is edema and chemosis of conjuctival, episcleral, and scleral tissue at initial presentation.

Abbreviations: OD, right eye; OS, left eye.

for a follow-up visit 4 days later with a reduction of both lid tenderness and pain on eye movement (Figure 4A and B). Red cap desaturation testing was equal in both eyes. The diffuse redness was still present, but the episcleral vessels were less dilated and no longer elevated. Due to the improvement in inflammation, discontinuation of oral prednisone and institution of oral NSAIDs therapy was considered. However, due to a history of stomach ulcers with Motrin ${ }^{\circledR}$ usage, prednisone was continued. The scheduled taper was as follows: $40 \mathrm{mg} \times 4$ days, $20 \mathrm{mg} \times 4$ days, $10 \mathrm{mg} \times 7$ days, and $5 \mathrm{mg} \times 7$ days before discontinuing completely after a total of 24 days.

One week after the 4-day follow-up, the patient had complete resolution of her signs and symptoms and resumed normal contact lens wear (Figure 5A and B).
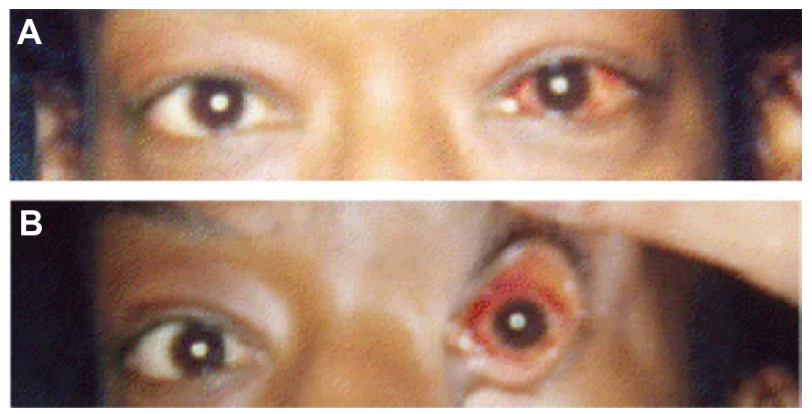

Figure 4 The patient at 4-day follow-up.

Notes: Note the improved droop of the left eye lid $(\mathbf{A})$ and the moderate reduction of redness and edema of sclera and episclera (B). 

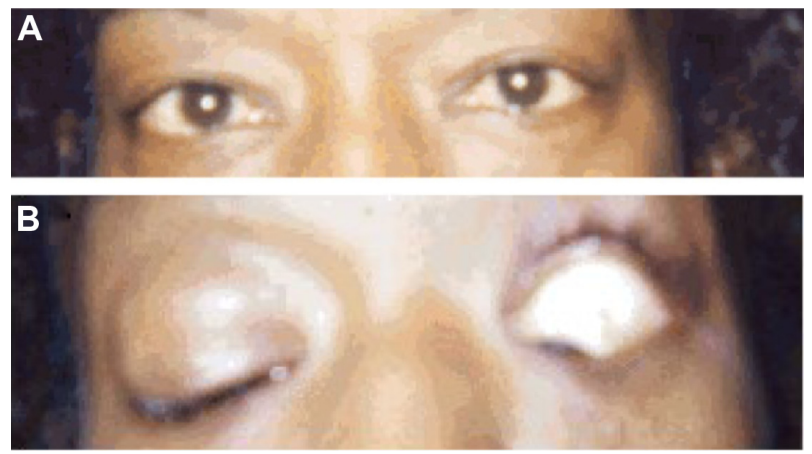

Figure 5 Complete resolution at 10 days.

Notes: Resolution of left eye lid droop was apparent after 10 days of oral steroid therapy $(\mathbf{A})$, and there was complete resolution of redness and edema of sclera and episclera with no observable scleral thinning after 10 days of oral steroid therapy (B).

The conjunctiva of the left eye was clear and quiet with no trace of dilated episcleral vessels or redness. There was no remaining edema of the sclera or episclera. The patient was still tapering oral prednisone as directed and had 2 weeks remaining until discontinuation was complete.

\section{Discussion}

Diffuse anterior scleritis is the most benign form of scleritis and occurs with the highest incidence in individuals during their fourth-seventh decades. ${ }^{1}$ Diffuse scleritis accounts for $40 \%-83 \%$ of scleritis cases. ${ }^{2}$ This type of inflammation usually presents at a younger age than the other types of scleritis and is equally prevalent between men and women., ${ }^{2,6}$ There is no known racial or geographic predilection. This contrasts with the predominantly Caucasian and female population among all forms of scleritis. ${ }^{7} \mathrm{Up}$ to $50 \%$ of all scleritis patients have an associated systemic disease. ${ }^{2,3}$ There is no known human leukocyte antigen association. ${ }^{7}$

\section{Clinical features and recognition}

As in the presented case, clinical features of scleritis include diffuse redness and a swollen episclera, which can obscure the sclera. In severe cases, the conjunctival edema may conceal the cornea and also protrude from between the eyelids. ${ }^{1}$ Occasionally only small or sectoral areas of the sclera may be affected. The term "brawny scleritis" applies to severe inflammation that is widespread with intense overlying injection, redness, and chemosis. ${ }^{2,8}$

Pain is the most characteristic symptom of scleritis, often prompting patients to seek eye care. ${ }^{1,79}$ The pain is boring in nature and most often localized to the eye. More rarely, the pain can be generalized from the orbital margin to the temple, sinuses, and jaw in the distribution of the trigeminal nerve. Such discomfort can also prevent patients from sleeping at night, especially in the necrotizing form. The pain is due to distention or destruction of the nerves passing through the sclera. ${ }^{8}$

Diffuse anterior scleritis is characterized by dilation of the superficial and deep anterior episcleral vascular plexuses, with distortion of the normal vascular pattern due to formation of large vascular channels in affected areas. ${ }^{1,2,8}$ The sclera is more commonly affected anterior to the equator because of the greater anterior vascular supply. Edema of the sclera and overlying episcleral tissue causes outward protrusion of the blood vessels and results in congestion of the scleral plexus. ${ }^{10}$ In the presented case, the patient had a definite protrusion of her blood vessels, and the sclera was bright red with a purplish hue. Unlike the presented case, after resolution, there can be subtle but permanent changes to the sclera. No scleral collagen is lost, but there is reorganization which increases scleral translucency to create a slightly bluish-red discoloration. Accompanying this change, is the alteration of the vasculature in the affected area that can cause vascular beading or focal, aneurysmal dilatations. ${ }^{2}$ The severity of inflammation may be directly associated with the amount of episcleral tissue present. ${ }^{8}$

Visualization of the scleral tissue can be achieved under different conditions, including under room lighting, behind the slit lamp, and with the red-free filter. Diffuse room lighting can determine if the superficial episcleral vessels are involved, versus the deeper scleral vessels. Slit lamp examination helps to confirm macroscopic findings by determining the depth of vascular involvement, infiltration, and edema. In addition, other ocular structures may be observed for evidence of keratitis, uveitis, and posterior segment changes. Red-free lighting can further assist with diagnosis by making the vascular alterations and vessels more apparent. ${ }^{1,7}$

\section{Systemic association}

Often scleritis can be the manifestation of an underlying systemic autoimmune disease. Less frequently it can be caused by infection. (Table 2), ${ }^{2,3}$ Studies indicate that $13 \%-45 \%$ of anterior scleritis patients have systemic disease. ${ }^{6,11,12}$ Rheumatoid arthritis is the most common systemic condition associated with scleritis. The incidence of rheumatoid arthritis in patients with scleritis ranges from $10 \%-33 \%$. Conversely, scleritis occurs in $0.15 \%-6.3 \%$ of patients with rheumatoid arthritis. ${ }^{13}$ Other associated autoimmune diseases include Wegener's granulomatosis, relapsing polychondritis, seronegative spondyloarthropathies (ankylosing spondylitis, Reiter's syndrome, psoriatic arthritis), inflammatory bowel disease (Crohn's disease and ulcerative colitis), systemic lupus erythematosus, and IgA nephropathy. ${ }^{12,14,15}$ 
Table 2 Scleritis-associated systemic diseases

\begin{tabular}{lll}
\hline Systemic diseases & Infectious diseases & Other \\
\hline Causes of scleritis & & \\
Rheumatoid arthritis & Syphilis & Ocular surgery \\
Wegener's granulomatosis & Sarcoidosis & Metabolic causes \\
Polyarteritis nodosa & Herpes zoster & Trauma \\
Relapsing polychondritis & Herpes simplex & \\
Psoriatic arthritis & Tuberculosis & \\
Reiter's syndrome & Lyme disease & \\
Ankylosing spondylitis & Bacterial & \\
Systemic lupus erythematosus & Fungal & \\
lgA nephropathy & HIV & \\
Giant cell arteritis & & \\
Behcet's disease & & \\
Takayasu's arteritis & & \\
Crohn's disease & & \\
Ulcerative colitis & & \\
Gout &
\end{tabular}

Abbreviation: HIV, human immunodeficiency virus.

Sainz de la Maza et $\mathrm{al}^{15}$ made comparisons of different types of anterior scleritis with and without systemic vasculitic disease. As with this case, they reported that diffuse anterior scleritis occurred with a greater frequency in patients without underlying systemic disease. ${ }^{15,16}$ Rheumatoid arthritis was the only systemic vasculitis associated with scleromalacia perforans, an asymptomatic thinning and necrosis of the sclera without inflammation. Rheumatoid arthritis is also the most frequently encountered disease in cases of bilateral scleritis, suggesting a more widespread distribution of extraarticular involvement. Nonrheumatoid scleritis is more often unilateral. Whether or not these patients have an autoimmune condition, this may represent a more localized disorder.

At the time of diagnosis, the presented patient did not have a known associated systemic disease. The patient was scheduled for further consultation with a rheumatologist to investigate her history of juvenile idiopathic arthritis, joint pains, and the most recent episode of anterior scleritis within the context of her laboratory results. The association of juvenile idiopathic arthritis and scleritis remains unclear, with the main ocular complications being uveitis, cataracts, and band keratopathy, whereas a definitive association with scleritis in adulthood remains unclear.

During follow-up in the rheumatology clinic, the patient was observed to have polyarticular arthralgias based on a history of scleritis, juvenile symptoms, and a positive ANA (1:160 nucleolar). The differential diagnoses included human leukocyte antigen-B27-associated arthritis, lupus-associated arthritis, seronegative rheumatoid arthritis, and recurrent juvenile idiopathic arthritis. Due to an incomplete work-up, the patient was tested for further serologies and was prescribed a low-dose steroid and Plaquenil (Sanofi Aventis, Bridgewater, NJ, USA) for early intervention based on her symptoms and presenting evidence. Of note was the positive ANA 1:160 nucleolar pattern, which is also suggestive of scleroderma and cannot be ruled out. Other conditions to consider with a positive ANA are systemic lupus erythematosus and Sjögren's syndrome. Systemic lupus erythematosus was a strong systemic possibility due to the positive ANA, but the patient denied any tightening of her skin, rash, or sicca symptoms. Lupus is more strongly correlated with the positive ANA speckled pattern, which she did not have, and the DNA testing for lupus by the rheumatology clinic was negative. Sjögren's syndrome testing for anti-Sjögren's syndrome A (SSA) and anti-Sjögren's syndrome B (SSB) antigens was also negative. Although the additional subserologies tested negative, the patient continued to endure joint pain, particularly in her wrists, and remains suspicious for possible lupus versus juvenile idiopathic arthritis based on her pattern of symptoms and presentation. She had a chest X-ray one month later and it was normal, which cleared her of any potential infection such as tuberculosis or sarcoidosis. After over 8 years of follow-up, no definitive systemic diagnosis has been yet determined, although the patient is still followed closely by the rheumatology clinic. She has not experienced a recurrence of scleritis.

Less common causes of scleritis include sarcoidosis, herpes zoster, herpes simplex, human immunodeficiency virus (HIV), Pseudomonas aeruginosa, tuberculosis, syphilis, gout, and ocular surgery. ${ }^{1,10,12}$ At least $5 \%-10 \%$ of anterior scleritis cases are of infectious origin. ${ }^{10}$ Although primarily a clinical diagnosis, histopathology has been shown to be able to differentiate the underlying cause of the scleritis. Scleritis associated with systemic autoimmune disease, including rheumatoid arthritis, will show a zonal granulomatous inflammation surrounding a central necrotic sclera, which supports an immune complex-mediated process for pathogenesis. Therefore, scleritis from rheumatoid arthritis versus the other systemic autoimmune diseases appears identical on pathology and cannot be differentiated from each other histopathologically. Idiopathic scleritis shows an absence of vasculitis and will have a nonzonal, diffuse scleral inflammation, suggesting a delayed type of hypersensitivity reaction. Infectious scleritis will exhibit necrosis with microabscesses. ${ }^{17}$

A recent study showed a $4 \%$ annual rate of developing a systemic disease in patients previously diagnosed with idiopathic scleritis. ${ }^{18}$ Fourteen percent of patients were diagnosed with systemic disease as a result of initial presentation of scleritis, and $8 \%$ had systemic disease diagnosed during follow-up. This suggests that patients diagnosed with scleritis 
should be worked-up at the time of diagnosis, in addition to undergoing periodic rheumatologic examination.

\section{Clinical examination and testing}

B-scan ultrasonography plays an important role in the diagnosis of scleritis by ruling out posterior involvement with the absence of scleral thickening or accompanying serous retinal detachment (Figure 2). Ultrasound biomicroscopy, which is a high-frequency form of ultrasound specifically for the anterior segment to provide high resolution of superficial structures, is a helpful tool in distinguishing scleritis from episcleritis based on thickness, reflectivity, and homogeneity of the sclera. ${ }^{19}$ Diffuse and nodular anterior scleritis will show scleral thickening, low reflectivity, and focal areas of hyporeflectivity. Ultrasound biomicroscopy also provides quantitative clinical measurements that allow for monitoring the clinical course of scleritis. ${ }^{20}$

The main differential diagnoses in the presented case were episcleritis, scleritis, and orbital inflammatory disease. Episcleritis will show blanching of the conjunctival vessels upon application of $10 \%$ phenylephrine, or $2.5 \%$ phenylephrine which is more widely available at eye care facilities, and patients do not experience the severity of ocular pain seen with scleritis. Additionally, areas of episcleritis will have salmon or pinkish color, whereas in scleritis, there will be more of a deep reddish-purple or bluish hue. ${ }^{1,9}$ Vision loss is not seen with episcleritis, and it usually responds quickly to topical corticosteroid treatment. One case series reported episcleritis converting to scleritis in two patients among the 37 subjects reviewed. ${ }^{21}$ In the presented case, the clinical signs and lack of vascular blanching allowed us to rule out episcleritis without the need for further testing.

Anterior segment fluorescein angiography in scleritis will demonstrate a normal conjunctival vascular pattern rapid flow rate, and a transit time of the dye is complete within 2-3 seconds. ${ }^{8}$ Unless there is the rare abnormal filling and leakage of deeper vessels, this is indistinguishable from the pattern seen in episcleritis. ${ }^{8,9}$ Indocyanine green angiography may be helpful in differentiating scleritis from episcleritis by demonstrating leakage that is specific to deep scleral inflammation and nearly absent in episcleritis. ${ }^{22}$ Other authors argue that the technique does not add any more useful clinical information than fluorescein angiography. ${ }^{23}$

\section{Orbital inflammatory disease versus scleritis}

Orbital inflammatory disease, the initial working diagnosis considered in the presented patient, can share overlapping features with scleritis. Orbital inflammatory disease is defined as a noninfective inflammation involving orbital tissues. It often occurs without an identifiable local or systemic cause. ${ }^{24}$ Orbital inflammatory disease may involve several orbital structures, or it can be localized to specific tissues such as the lacrimal gland (dacryoadenitis), extraocular muscles (myositis), posterior portion of the globe (periscleritis), or optic nerve sheath (optic perineuritis). ${ }^{25-27}$ Clinical characteristics can include conjunctival injection, orbital pain, proptosis, and often, altered eye movements. There can be associated swelling and erythema of the eyelids. Reported systemic immunological disorders associated with orbital inflammatory disease include Crohn's disease, systemic lupus erythematosus, rheumatoid arthritis, myasthenia gravis, and ankylosing spondylitis. ${ }^{28}$ Spontaneous resolution is possible, although vision loss and oculomotor dysfunction may result without medical intervention. ${ }^{29}$ The orbital structures involved can be delineated by MRI. Systemic corticosteroids remain the established therapeutic treatment of choice. Given the patient's presentation of moderate to severe pain upon eye movement, eyelid involvement, and mild color vision deficit, orbital inflammatory disease was ruled out but was a potential working diagnosis versus scleritis. Once the MRI results were determined to be normal on the following day, the patient's diagnosis was confirmed as scleritis. Occasionally, scleritis and orbital inflammatory disease can both be present. Boonman et $\mathrm{al}^{29}$ investigated an association between all forms of scleritis with myositis in a retrospective, noncomparative case series. Of 103 patients with a diagnosis of scleritis or episcleritis, orbital myositis was found to be present in eleven patients. Ocular complications were more common in $64 \%$ of patients with combined scleritis and myositis compared to $30.4 \%$ of patients with scleritis alone. This indicates a more diffuse and severe inflammation of scleritis in the presence of myositis, although the prognosis for vision is overall unaffected.

\section{Treatment options}

Treatment for diffuse anterior scleritis often involves systemic medications, including NSAIDs, corticosteroids, or immunosuppressive therapy ${ }^{21,30}$ (Table 3). Patients are started on oral NSAIDs as the initial treatment of choice for diffuse and nodular scleritis unless there are contraindications. ${ }^{14}$ Recommended treatments include indomethacin $75 \mathrm{mg}$ twice a day or $25 \mathrm{mg}$ four times a day, with reduced dosing as scleritis resolves. ${ }^{14,30}$ Nonresponsive or contraindicated patients may be placed on oral prednisone $2 \mathrm{mg} / \mathrm{kg}$ daily, with tapering. 
Table 3 Treatment modalities for scleritis and episcleritis, with drugs and dosages

\begin{tabular}{ll}
\hline Scleritis: & Episcleritis: \\
Topical and/or oral NSAIDs & Artificial tears \\
Topical and/or oral steroids & Topical NSAIDs \\
Immunosuppressive therapy & Topical steroids \\
Subconjunctival corticosteroid & Topical and/or oral NSAIDs \\
injections & Oral NSAIDs: \\
Topical NSAIDs: & Ibuprofen $400-600 \mathrm{mg}$ qid \\
Nepafenac qid & Naproxen $375-500 \mathrm{mg}$ bid \\
Ketorolac tromethamine qid & Indomethacin $75 \mathrm{mg}$ bid \\
Diclofenac sodium qid & Flurbiprofen $100 \mathrm{mg}$ tid \\
Bromfenac qid & Piroxicam $20 \mathrm{mg}$ qd \\
Suprofen qid & Diflunisal $500 \mathrm{mg}$ bid \\
Flurbiprofen qid & Sulindac $200 \mathrm{mg}$ bid \\
Topical steroids: & Oral steroids: \\
Prednisolone acetate qid & Prednisone $60-120 \mathrm{mg}$ bid for \\
Fluorometholone qid & first week with rapid tapering \\
Dexamethasone qid & to $20 \mathrm{mg}$ daily within \\
Immunosuppressive therapy: & $2-3$ weeks, slow taper \\
Cyclosporine 2-5 mg/kg & by $2.5 \mathrm{mg}$ steps \\
orally daily & Subconjunctival corticosteroid \\
Cyclophosphamide, oral & injections: \\
I-2 mg/kg daily & Triamcinolone acetonide \\
Methotrexate $7.5-15 \mathrm{mg}$ orally or & $0.25-1.0 \mathrm{~mL}$ of $40 \mathrm{mg} / \mathrm{mL}$ \\
I5 mg intramuscularly I $\times /$ week & suspension \\
Azathioprine I-2 mg/kg orally daily & \\
\hline Abbrevition & \\
\hline
\end{tabular}

Abbreviations: bid, two times a day; NSAIDs, nonsteroidal anti-inflammatory drugs; qd, one time a day; qid, four times a day; tid, three times a day.

Third-line therapy includes immunosuppressive drugs such as cyclophosphamide; patients are started on $2 \mathrm{mg} / \mathrm{kg} / \mathrm{day}$ while taking oral prednisone. ${ }^{10,30}$ Other immunosuppressive agents include methotrexate, azathioprine, cyclosporine, and chlorambucil, but their effectiveness in scleritis has not been well-documented. ${ }^{10}$ In contrast, immunosuppressive drugs are first line therapy in cases of necrotizing scleritis. ${ }^{14}$ Patients with any form of anterior scleritis and who smoke during treatment will have a delayed recovery response compared to nonsmokers, independent of treatment type and may therefore require more intensive therapy. ${ }^{31}$

Topical anti-inflammatory therapy is another treatment option for diffuse anterior scleritis. McMullen et $\mathrm{al}^{32}$ reported a $47 \%$ success rate for nodular and diffuse anterior scleritis while using topical Pred Forte ${ }^{\circledR}$ (Irvine, CA, USA) (prednisolone acetate) ophthalmic suspension 1\% during a 2 month treatment period. They recommended that topical steroid therapy can be considered as first-line treatment for nonnecrotizing anterior scleritis, especially for patients who are prone to systemic side effects of medical therapy. ${ }^{32}$ Due to the severity of ocular inflammation in the presented case, oral therapy was determined to be necessary.
Controversy exists regarding the use of subconjunctival corticosteroid injections for nonnecrotizing, noninfectious anterior scleritis. Originally, it was pronounced contraindicated due to concern of scleral thinning and perforation, ${ }^{8,9,33}$ but successful treatments have been reported. ${ }^{34-40}$ Subconjunctival corticosteroid injections offer the advantages of providing an immediate effect, being well-tolerated, and avoiding the need to treat patients with systemic steroid or immunosuppressive therapy with associated side effects.

\section{Complications and prognosis}

Elevated intraocular pressure can occur in any form of anterior or posterior scleritis, with secondary glaucoma developing in $9 \%-22 \%{ }^{1,6}$ The increased intraocular pressure may also be attributed to acute congestion of outflow channels, raised episcleral venous pressure, or it may be a steroid-induced rise. ${ }^{1,10}$

Vision loss from anterior scleritis usually results from cataract formation, glaucoma, uveitis, corneal involvement, or posterior segment disease. Keratitis can lead to stromal thinning and subsequent scarring with chronic inflammation resulting in neovascularization. ${ }^{1,8}$ Cataracts are more frequent among the necrotizing form of anterior scleritis, with an approximate incidence of only $5 \%$ among diffuse anterior scleritis. ${ }^{1}$

The ocular prognosis of anterior scleritis varies with different systemic vasculitic diseases. Scleritis in patients with systemic vasculitis carries a worse visual prognosis than in patients without systemic vasculitic disease. Scleritis occurring in spondyloarthropathies or systemic lupus erythematosus is often self-limited and seldom with visual complications, whereas Wegener's granulomatosis is a more severe condition, with sight-threatening consequences including blindness due to progression to necrotizing scleritis, with resultant perforation and phthisis bulbi in advanced cases. ${ }^{15}$ Scleritis concurrent with rheumatoid arthritis or relapsing polychondritis is of moderate severity and visual threat, requiring close monitoring and follow up. ${ }^{13}$ Recurrence is most common in patients with anterior diffuse scleritis than any other form. ${ }^{1}$ The recurrences are more severe with each episode. Up to $25 \%$ of diffuse anterior scleritis can progress to nodular or necrotizing forms upon repeated attacks. ${ }^{3}$ Tuft and Watson ${ }^{6}$ reported an overall observation of $35.9 \%$ recurrence of scleritis in a study of 290 patients diagnosed with the different forms of scleritis, where $22.8 \%$ had a recurrence of the same form of scleritis, and the other $13.1 \%$ had a recurrence of a different type of scleritis. 


\section{Conclusion}

Even with prompt and aggressive treatment, anterior scleritis can be severe enough to lead to loss of the eye, occasionally requiring enucleation as relief from unbearable pain. In cases of anterior scleritis, where response to therapy is effective, a high index of clinical suspicion should remain regarding investigation for a systemic association. Fortunately, the patient in the presented case had complete resolution and no visual consequences. Full rheumatologic evaluation has yet to discover a definitive associated systemic disease, although the patient remains under continuous follow-up care and monitoring.

\section{Disclosure}

The authors report no conflicts of interest in this work.

\section{References}

1. Watson PG, Hayreh SS. Scleritis and episcleritis. Br J Ophthalmol. 1976;60(3):163-191.

2. McCluskey PJ, Wakefield D. Scleritis and episcleritis. In: Pepose JS, Holland GN, Wilhelmus KR, editors. Ocular Infection and Immunity. St Louis, MO, USA: Mosby; 1996:642-647.

3. Benson WE. Posterior scleritis. Surv Ophthalmol. 1988;32(5):297-316.

4. McCluskey PJ, Watson PG, Lightman S, Haybittle J, Restori M, Branley M. Posterior scleritis: clinical features, systemic associations and outcome in a large series of patients. Ophthalmol. 1999;106:2380-2386.

5. Purvin V, Kawasaki A, Jacobson DM. Optic perineuritis: clinical and radiographic features. Arch Ophthalmol. 2001;119:1299-1306.

6. Tuft SJ, Watson PG. Progression of scleral disease. Ophthalmology. 1991;98(4):467-471.

7. Okhravi N, Odufuwa B, McCluskey P, Lightman S. Scleritis. Surv Ophthalmol. 2005;50(4):351-363.

8. Watson P. Diseases of the sclera and episclera. In: Tasman W, Jaeger EA, editors. Duane's Clinical Ophthalmology. Philadelphia, PA: JB Lippincott; 2005: 1-45.

9. Watson PG. Doyne Memorial Lecture, 1982. The nature and treatment of scleral inflammation. Trans Ophthalmol Soc U K. 1982;102(pt 2): 257-281.

10. Albini TA, Rao NA, Smith RE. The diagnosis and management of anterior scleritis. Int Ophthalmol Clin. 2005;45(2):191-204.

11. Sainz de la Maza M, Jabbur NS, Foster CS. Severity of scleritis and episcleritis. Ophthalmology. 1994;101(2):389-396.

12. Raiji VR, Palestine AG, Parver DL. Scleritis and systemic disease association in a community-based referral practice. Am J Ophthalmol. 2009;148(6):946-950.

13. McGavin DD, Williamson J, Forrester JV, et al. Episcleritis and scleritis: a study of their clinical manifestations and association with rheumatoid arthritis. Br J Ophthalmol. 1976;60(3):192-226.

14. Sainz de la Maza M, Jabbur NS, Foster CS. An analysis of therapeutic decision for scleritis. Ophthalmology. 1993;100(9):1372-1376.

15. Sainz de la Maza M, Foster CS, Jabbur NS. Scleritis associated with systemic vasculitic diseases. Ophthalmology. 1995;102(4):687-692.

16. Sainz de la Maza M, Foster CS, Jabbur NS. Scleritis associated with rheumatoid arthritis and with other systemic immune-mediated diseases. Ophthalmology. 1994;101(7):1281-1288; discussion 1287-1288.

17. Riono WP, Hidayat AA, Rao NA. Scleritis: a clinicopathologic study of 55 cases. Ophthalmology. 1999;106:1328-1333.
18. Akpek EK, Thorne JE, Qazi FA, Do DV, Jabs DA. Evaluation of patients with scleritis for systemic disease. Ophthalmology. 2004;111(3): 501-506.

19. Heiligenhaus A, Schilling M, Lung E, Steuhl KP. Ultrasound biomicroscopy in scleritis. Ophthalmology. 1998;105(3):527-534.

20. Pavlin CJ, Easterbrook M, Hurwitz JJ, Harasiewicz, Eng P, Foster FS. Ultrasound biomicroscopy in the assessment of anterior scleral disease. Am J Ophthalmol. 1993;116(5):628-635.

21. Jabs DA, Mudun A, Dunn JP, Marsh MJ. Episcleritis and scleritis: clinical features and treatment results. Am J Ophthalmol. 2000;130(4): 469-476.

22. Guex-Crosier Y, Durig J. Anterior segment indocyanine green angiography in anterior scleritis and episcleritis. Ophthalmology. 2003;110(9): $1756-1763$.

23. Stanga PE, Lim JI, Hamilton P. Indocyanine green angiography in chorioretinal diseases: indications and interpretation: and evidencebased updtate. Ophthalmology. 2003;110(1):15-21; quiz 22.

24. Yuen SJ, Rubin PAD. Idiopathic orbital inflammation: ocular mechanisms and clinicopathology. Ophthalmol Clin NAm. 2002;15(1):121-126.

25. Gordon LK. Diagnostic dilemmas in orbital inflammatory disease. Ocular Immunol Inflamm. 2003;11(1):3-15.

26. Kennerdell JS, Dresner SC. The nonspecific orbital inflammatory syndromes. Surv Ophthalmol. 1984;29(2):93-103.

27. Sekhar GC, Mandal AK, Vyas P. Non-specific orbital inflammatory disease. Doc Ophthalmol. 1993;84(2):155-170.

28. Yuen SJ, Rubin PA. Idiopathic orbital inflammation: distribution, clinical features, and treatment outcome. Arch Ophthalmol. 2003; 121(4):491-499.

29. Boonman ZF, de Keizer RJ, Graniewski-Wijnands HS, Watson PG. Orbital myositis in scleritis. Br J Ophthalmol. 2003;87(1):38-42.

30. Rachitskaya A, Mandelcorn ED, Albini TA. An update on the cause and treatment of scleritis. Curr Opin Ophthalmol. 2010;21(6):463-467.

31. Boonman ZF, de Keizer RJ, Watson PG. Smoking delays the response to treatment in episcleritis and scleritis. Eye (Lond). 2005;19(9): 949-955.

32. McMullen M, Kovarik G, Hodge WG. Use of topical steroid therapy in the management of nonnecrotizing anterior scleritis. Can J Ophthalmol. 1999;34(4):217-221.

33. Watson PG. Treatment of scleritis and episcleritis. Trans Ophthalmol Soc U K. 1974;94:76-79.

34. Croasdale CR, Brightbill FS. Subconjunctival corticosteroid injections for nonnecrotizing anterior scleritis. Arch Ophthalmol. 1999;117(7): 966-968.

35. Tu EY, Culbertson WW, Pflugfelder SC, Huang A, Chodosh JC. Therapy of nonnecrotizing anterior scleritis with subconjunctival corticosteroid injection. Ophthalmology. 1995;102(5):718-724.

36. Zamir E, Read RW, Smith RE, Wang RC, Rao NA. A prospective evaluation of subconjunctival injection of triamcinolone acetonide for resistant anterior scleritis. Ophthalmology. 2002;109(4):798-805; discussion 805-807.

37. Albini TA, Zamir E, Read RW, Smith RE, See RF, Rao NA. Evaluation of subconjunctival triamcinolone for nonnecrotizing anterior scleritis. Ophthalmology. 2005;112(10):1814-1820.

38. Sen HN, Ursea R, Nussenblatt RB, Buggage RR. Subconjunctival corticosteroid injection for the treatment of non-necrotising anterior scleritis. Br J Ophthalmol. 2005;89(7):917-918.

39. Johnson KS, Chu DS. Evaluation of sub-tenon triamcinolone acetonide injections in the treatment of scleritis. Am J Ophthalmol. 2010;149(1): $77-81$.

40. Roufas A, Jalaludin B, Gaskin C, McCluskey P. Subconjunctival triamcinolone treatment for non-necrotising anterior scleritis. $\mathrm{Br} \mathrm{J}$ Ophthalmol. 2010;94(6):743-747. 


\section{Publish your work in this journal}

Clinical Optometry is an international, peer-reviewed, open access journal publishing original research, basic science, clinical and epidemiologica studies, reviews and evaluations on clinical optometry. All aspects of patient care are addressed within the journal as well as the practice of optometry including economic and business analyses. Basic and clinical

Submit your manuscript here: http://www.dovepress.com/clinical-optometry-journal

\section{Dovepress}

research papers are published that cover all aspects of optics, refraction and its application to the theory and practice of optometry. The manuscript management system is completely online and includes a very quick and fair peer-review system, which is all easy to use. Visit http://www.dovepress. com/testimonials.php to read real quotes from published authors. 